\title{
Transcriptome and alternative splicing analysis of nucleus pulposus cells in response to high oxygen tension: Involvement of high oxygen tension in the pathogenesis of intervertebral disc degeneration
}

\author{
CHENCHENG FENG, YANG ZHANG, MINGHUI YANG, MINGHONG LAN, \\ BO HUANG, HUAN LIU and YUE ZHOU \\ Department of Orthopedics, Xinqiao Hospital, The Third Military Medical University, \\ Chongqing 400037, P.R. China
}

Received January 11, 2017; Accepted February 22, 2018

DOI: $10.3892 / \mathrm{ijmm} .2018 .3523$

\begin{abstract}
High oxygen tension caused by neovascularization in the microenvironment of intervertebral discs (IVDs) is associated with the pathogenesis of IVD degeneration (IDD). Pre-mRNAs undergo alternative splicing (AS) to produce structurally and functionally diverse mRNA and proteins. However, the precise role of high oxygen tension in IDD and the relationship between AS and high oxygen tension in disc cells remain unknown. To investigate the effect of high oxygen tension on disc cells, Affymetrix Rat Transcriptome Array 1.0 was used to determine differentially expressed genes (DEGs) and alternative splicing genes (ASGs) in rat nucleus pulposus (NP) cells treated with $20 \% \mathrm{O}_{2}$. NP cells at $1 \% \mathrm{O}_{2}$ served as the control. PCR was used for validation. GO and KEGG pathway analysis was performed. Furthermore, the reactive oxygen species (ROS) production, growth, cell cycle and matrix metabolism of NP cells were also investigated. In
\end{abstract}

Correspondence to: Dr Huan Liu or Professor Yue Zhou, Department of Orthopedics, Xinqiao Hospital, The Third Military Medical University, 83 Zheng Street, Chongqing 400037, P.R. China

E-mail: doctorfgy@163.com

E-mail: happyzhou@vip.163.com

Abbreviations: IVD, intervertebral disc; IDD, intervertebral disc degeneration; LBP, low back pain; NP, nucleus pulposus; $\mathrm{AF}$, annulus fibrosus; AS, alternative splicing; ECM, extracellular matrix; Nox4, NADPH oxidase 4; DEG, differentially expressed gene; ASG, alternative splicing gene; SI, splicing index; ADAMTS, a disintegrin and metalloproteinase with thrombospondin motifs; MMP, matrix metalloproteinase; BMP, bone morphogenetic protein; ROS, reactive oxygen species; GAS1, growth arrest specific 1; VEGFA, vascular endothelial growth factor A; GPX1, glutathione peroxidase 1; HIF-1, hypoxia inducible factor-1; PBS, phosphate-buffered saline; ASE, alternative splicing exon

Key words: intervertebral disc degeneration, oxidative stress, alternative splicing, extracellular matrix metabolism, cell cycle arrest, transcriptome total, 2499 DEGs and 8451 ASGs were identified. Various GO terms and KEGG pathways were potently associated with IDD, including autophagy, mTOR signaling pathway and angiogenesis. Especially, high oxygen tension increased ROS production in NP cells. It also accelerated the matrix metabolism of NP cells and induced NP cell cycle arrest to retard cell growth. This study, for the first time, analyzes the transcriptome and AS of NP cells in response to high oxygen tension, indicating that high oxygen tension is involved in the establishment and progression of IDD through its wide effects on the viability and function of disc cells.

\section{Introduction}

Intervertebral disc (IVD) degeneration (IDD) is widely known as a main contributor to low back pain (LBP) that is prevalent worldwide $(1,2)$. The etiological factors of IDD involve aging, smoking, infection, abnormal mechanical stress, diabetes, trauma and genetic predisposition (3-8). Degenerative discs are structurally characterized by disc space collapse, nucleus pulposus (NP) dehydration, annulus fibrosus (AF) fissures and cartilage endplate calcification. Consideration of massive socio-economic burdens caused by IDD-associated LBP, elucidating the causes and pathogenesis of IDD in detail benefits developing new measures for the prevention and treatment of LBP.

The pathogenesis of IDD is complicated. The microenvironment of IVDs is hypoxic but not entirely anaerobic $\left(1 \% \mathrm{O}_{2}\right.$ in central NP) $(9,10)$. Resident disc cells survive well in this hypoxic microenvironment and still have oxygen-consumption metabolic processes (11-13). With IDD progression, neovascularization in discs increases oxygen tension in the microenvironment of IVDs $(14,15)$. High oxygen tension is expected to enhance reactive oxygen species (ROS) production and subsequently to cause oxidative stress in the microenvironment of IVDs, which is strongly associated with the establishment and progression of IDD (16-18). However, the role of high oxygen tension in the pathogenesis of IDD remains to be a relatively poorly explored area. Furthermore, IDD is a disc cell-mediated pathological 
process $(19,20)$. The initiation and progression of IDD depend on the viability and function of disc cells. Although high oxygen tension has been shown to reinforce the matrix catabolism and autophagy of NP cells $(21,22)$, the effect of high oxygen tension on the viability and function of disc cells and the molecular mechanism underlying the effect should be investigated further in depth to elucidate the involvement of high oxygen tension in the pathogenesis of IDD.

Alternative splicing (AS) is a regulatory process by which the exons of pre-mRNAs are spliced in different ways to synthesize structurally and functionally diverse mRNA and proteins. Approximately $>90 \%$ of multiexonic genes undergo AS regulation. It is crucial to increasing the macromolecular and cellular complexity of eukaryotic organisms without extending genome size (23-25). The types of AS that are responsible for RNA isoform generation includes cassette alternative exon (exon skip or inclusion), intron retention, alternative $5^{\prime} / 3^{\prime}$ splice sites, mutually exclusive alternative exons, alternative promoter and first exon as well as alternative poly-A site and terminal exon. Among them, cassette alternative exon is the most common AS event and has been investigated extensively (25). AS has been reported to be involved in various diseases, including autoimmune disease, neurodegenerative disease and carcinoma (26-28). With respect to IDD, the AS of fibronectin, thrombospondins and versican has been determined to be associated with matrix remodeling of degenerative discs (29-31). However, the role of AS in the pathogenesis of IDD remains unclear. Besides, AS probably is an essential mechanism mediating the effect of high oxygen tension on disc cells.

In the present study, in order to investigate the transcriptome and AS of disc cells in response to high oxygen tension, rat NP cells were cultured at $1 \% \mathrm{O}_{2}$, and then were treated with $20 \% \mathrm{O}_{2}$ (high oxygen tension). After that, total RNA of NP cells was extracted to perform microarray assays using the Affymetrix Rat Transcriptome Array 1.0. A comparative analysis between NP cells at $1 \% \mathrm{O}_{2}$ and NP cells treated with high oxygen tension was performed to determine differentially expressed genes (DEGs) and alternative splicing genes (ASGs). The function of DSGs and ASGs was annotated through Gene Ontology (GO) analysis and Kyoto Encyclopedia of Genes and Genomes (KEGG) pathway analysis. Moreover, increased ROS production induced by high oxygen tension was observed in NP cells. We also analyzed the growth, cell cycle distribution and matrix metabolism of NP cells to support the results of microarray further. Our study revealed the functional role of high oxygen tension in NP cells on genome-wide scale. Also, we investigated the regulatory effect of high oxygen tension on AS of NP cells, suggesting the involvement of AS in the pathogenesis of IDD. This study contributes to understanding the regulatory effect of high oxygen tension on NP cells, which gives a novel insight into the establishment and progression of IDD.

\section{Materials and methods}

Ethics statement. This study was approved by the Ethics Committee of Xinqiao Hospital, Third Military Medical University. All experimental procedures described in this study were in accordance with the standards set forth in the eighth edition of Guide for the Care and Use of Laboratory
Animals published by the National Academy of Sciences (The National Academies Press, Washington, DC, USA).

$\mathrm{NP}$ cell culture at $1 \% \mathrm{O}_{2}$ and high oxygen tension $\left(20 \% \mathrm{O}_{2}\right)$ treatment. NP tissues were harvested from caudal discs (C1-C10) of adult (3-month-old) male Sprague-Dawley rats (Laboratory Animal Research Center of Daping Hospital, Chongqing, China) after sacrificed using excessive pentobarbital sodium. The isolated tissues were incubated with Dulbecco's modified Eagle's medium (DMEM)/F-12 (Invitrogen, Carlsbad, CA, USA) containing $0.2 \%$ type II collagenase (Sigma, St. Louis, MO, USA) at $37^{\circ} \mathrm{C}$ and $1 \% \mathrm{O}_{2}$ for $2 \mathrm{~h}$. A $70 \mu \mathrm{m}$ cell mesh was used to remove tissue debris. After centrifuging at $1,000 \mathrm{rpm}$ for $5 \mathrm{~min}$, the supernatant of cell suspension was removed followed by resuspension with DMEM/F12 containing 10\% fetal bovine serum and 1\% penicillin/streptomycin (Invitrogen). NP cells grew as monolayer in $25 \mathrm{~cm}^{2}$ culture flasks (Corning, Inc., Corning, NY, USA) at $37^{\circ} \mathrm{C}$ and $1 \% \mathrm{O}_{2}$. Culture medium was changed twice a week. When confluent, NP cells were sub-cultured. The second or third culture passages were used in this study. For high oxygen tension treatment, NP cells were transferred into an incubator with $20 \% \mathrm{O}_{2}$ at $37^{\circ} \mathrm{C}$ for 7 days.

RTA 1.0 and bioinformatics analysis. NP cells without high oxygen tension treatment served as the control. The high oxygen tension-treated samples $(n=3)$ and the control samples $(n=3)$ lysed by TRIzol (Takara Bio, Shiga, Japan) were sent to Bioassay Laboratory of CapitalBio Corp. (Beijing, China). The global gene expression profile and alternative splicing exons (ASEs) of NP cells were analyzed using RTA 1.0 (Affymetrix Corp., Santa Clara, CA, USA). The hybridization, scanning and data extraction of microarray were performed in Bioassay Laboratory of CapitalBio Corp. according to the protocol provided by Affymetrix Corp. Concisely, the fluorescence signals of microarray scanned as DAT files were transformed into CEL files via the AGCC software (Affymetrix Corp.). Then, Affymetrix Expression Console software pretreated CEL files through robust multichip analysis algorithm to obtain chp files (32). The chp files were analyzed by Affymetrix Transcriptome Analysis Console software to determine DEGs and ASGs. DEGs were identified according to fold changes (fold change $\geq 2$ or $\leq-2)$ and $p$-value $(\mathrm{p}<0.05)$. ASGs were identified according to splicing index (SI, SI $\geq 2$ or $\leq-2)$ and $p$-value $(\mathrm{p}<0.05)$. SI is the ratio of the exon signal intensities normalized to the gene signal intensities between the high oxygen tension-treated group and the control group. It generally represents the exon exclusion/inclusion level $(33,34)$. Herein, $\mathrm{SI} \geq 2$ is regarded as general exon inclusion while $\mathrm{SI} \leq-2$ is regarded as general exon exclusion. GO analysis (http://www.geneontology.org) and KEGG pathway analysis (http://www.genome.jp/kegg) were performed to identify the cellular components, molecular functions, biological processes and signaling pathways enriched by DEGs or ASGs.

Reversed transcription-quantitative PCR (RT-qPCR) and semi-quantitative RT-PCR. Total RNA of NP cells were extracted using TRIzol reagent. The concentration of RNA was measured using a NanoDrop ND-2000 spectrophotometer (Thermo Fisher Scientific, Waltham, MA, USA). One micro- 
Table I. Primer sequences used in semi-quantitative RT-PCR analysis.

\begin{tabular}{lll}
\hline ASG (Exon) & \multicolumn{1}{c}{ Forward primer } & \multicolumn{1}{c}{ Reverse primer } \\
\hline ADAMTS9 (Exon 31) & CTACAGGCAAAGGCTGGTCTCC & CAGGACAGTCCCTCAGGTAGCA \\
FNDC1 (Exon 11) & GCAATCGGTCTTCGCTGAGGAG & GCATCGGTGTGGTGGAGGAGTA \\
GDF15 (Exon 2) & GCTGCTGTCACCTGGAGACTGT & GCCGCTGTCTGTCCTGTGCATA \\
KRCC1 (Exon 3) & GAGTTTGATTCCAGGCCCAGGT & CTGCCTTTCTCTGCCCTCCTCT \\
NPTN (Exon 2) & GGGTTTGTCAAGTCGCCCATGT & TCAGCACACTCACGCCGTTG \\
OGN (Exon 8) & TGGTTGTAGCAGACCGCCATCT & GGGAAGGTCACTGGGAGCACTT \\
PREB (Exon 4) & TGGTTACTGTGGGCTGGGACTT & CCTGGTAGCGGTACGGTGTGTT \\
SEC31A (Exon 23) & ACCAGCCAGCCCAGCAGTATT & TTCCAGGAGGCAGTGCATAAGC \\
TJP1 (Exon 27) & GCAGAAGCCTCATCTCCAGTCC & GCGACGGCAATGACACTCCTT \\
GAPDH & GTCCATGCCATCACTGCCACTC & GATGACCTTGCCCACAGCCTTG \\
\hline
\end{tabular}

ASG, alternative splicing gene; ADAMTS9, a disintegrin and metalloproteinase with thrombospondin motifs 5; FNDC1, fibronectin type III domain containing 1; GDF15, growth and differentiation factor 15; KRCC1, lysine-rich coiled-coil 1; NPTN, neuroplastin; OGN, osteoglycin; PREB, prolactin regulatory element binding; SEC31A, SEC31 homolog A, COPII coat complex component; TJP1, tight junction protein 1.

Table II. Primer sequences used in the RT-qPCR analysis.

\begin{tabular}{lll}
\hline Target gene & \multicolumn{1}{c}{ Forward primer } & \multicolumn{1}{c}{ Reverse primer } \\
\hline MMP13 & TACGAGCATCCATCCCGAGACC & TGAACCGCAGCACTGAGCCT \\
BMP3 & AGCCTTCAGACTCAGCCTCCTG & TCGCCTCGCCTTCTTCAGTGT \\
BMP4 & GACTTCGAGGCGACACTTCTGC & GGTTCCTGGCTCTGCTCTTCT \\
Neuropilin 1 & TCGGTGGGATTGCTGTGGATGA & TGCCTGGCTTCCTGGAGATGTT \\
ADAMTS5 & GCTCCTCTTGGTGGCTGACTCT & GCGTTCTTGCTCACCTCCAGAC \\
p15 & GGCTTCCTGGACACGCTAATGG & ATATCACGGTGGCCCTGCTCTT \\
GAS1 & TTTCTGCTGCTCCTGCTGCTTG & GGGTCAGTGCTCCCGATCATCT \\
Nox4 & CGCACAGTCCTGGCTTACCTTC & GGCAGCTACATGCACACCTGAG \\
PCNA & CGCAACTCCGCCACCATGTT & TTCACGCCGCCCGAACTGAT \\
VEGFA & TCACCACCACACCACCATCGT & ATCCAGTTCCACGAGGGACCAC \\
Cyclin I & AGCAGCCTTCCACCTCCATCTC & AGCCGCTTGATCCCGTCATACA \\
GDF15 & CCTGCTGTTCCTGCTGCTCTTG & TAGCTCGTCCGGGTTGAGTTGG \\
GPX1 & AGGCTCACCCGCTCTTTACCTT & TGGAACACCGTCTGGACCTACC \\
Aggrecan & ATCCGCTGCTCCAGAAGTGAGT & ACGGTGGTGCTGACGGTAACA \\
Collagen type II & CATGAACGGCGGCTTCCACTT & GCTTCGTCCAGGTAGGCAATGC \\
HIF-1 & CGCAACTGCCACCACTGATGAA & GGCTGTCCGACTGTGAGTACCA \\
GAPDH & GTCCATGCCATCACTGCCACTC & GATGACCTTGCCCACAGCCTTG \\
\hline
\end{tabular}

MMP13, matrix metalloproteinase 13; ADAMTS5, a disintegrin and metalloproteinase with thrombospondin motifs 5; BMP, bone morphogenetic protein; Nox4, nicotinamide adenine dinucleotide phosphate (NADPH) oxidase 4; GAS1, growth arrest specific 1; PCNA, proliferating cell nuclear antigen; VEGFA, vascular endothelial growth factor A; GDF15, growth and differentiation factor 15; GPX1, glutathione peroxidase 1 ; HIF-1, hypoxia inducible factor- 1 .

gram of RNA was reversely transcribed into cDNA using a PrimeScript RT reagent kit (Takara Bio) according to the protocol provided by the manufacturer. For semi-quantitative RT-PCR, $1 \mu \mathrm{l}$ cDNA was used for each reaction. The primers of target exons were designed based on constitutively expressed exons flanking the target exons (Table I). Glyceraldehyde 3 -phosphate dehydrogenase GAPDH served as the internal reference gene. The representative ASEs for semi-quantitative RT-PCR validation were selected according to three criteria: i) $|\mathrm{SI}|>3$, ii) cassette alternative exon and iii) for the feasibility of primer design, not the first and the last alternative exons. On the other hand, a ViiA ${ }^{\mathrm{TM}} 7$ Real-Time PCR system (Applied Biosystems, Thermo Fisher Scientific) was used to perform real-time quantitative PCR in triplicate. Twenty microliters SYBR $^{\circledR}$ Premix Ex Taq ${ }^{\mathrm{TM}}$ II (Takara Bio) reaction volume was composed of $10 \mu 1 \mathrm{SYBR}, 6 \mu 1 \mathrm{H}_{2} \mathrm{O}, 0.4 \mu \mathrm{l} \mathrm{ROX}, 0.8 \mu \mathrm{l}$ forward primer, $0.8 \mu 1$ reverse primer and $2 \mu \mathrm{l}$ cDNA. The cycle parameters were $95^{\circ} \mathrm{C}$ for $30 \mathrm{sec}$, followed by 40 cycles of $95^{\circ} \mathrm{C}$ for $5 \mathrm{sec}$ and $60^{\circ} \mathrm{C}$ for $30 \mathrm{sec}$. The relative fold changes of target genes were analyzed according to the $2^{-\Delta \Delta \mathrm{Ct}}$ algorithm (35). The internal reference gene was GAPDH. The primers of target genes used in this study are listed in Table II. 
ROS detection. 2',7'-Dichlorfluorescein-diacetate (DCFH-DA) is oxidized by ROS to generate dichlorofluorescein (DCF) with high fluorescence. Therefore, ROS production in NP cells was detected using DCFH-DA (Sigma). NP cell suspension was incubated with $\mathrm{H} 2 \mathrm{DCF}-\mathrm{DA}(25 \mu \mathrm{M})$ dissolved in phosphate-buffered saline (PBS) at $37^{\circ} \mathrm{C}$ and $5 \% \mathrm{CO}_{2}$ for $30 \mathrm{~min}$ followed by washing with serum-free DMEM/F12 three times. The mean fluorescence intensity (MFI) of DCF in NP cells was detected using a flow cytometer (Beckman Coulter, Inc., Brea, CA, USA).

Western blot analysis. NP cell proteins were extracted using the extraction reagent (Thermo Fisher Scientific). Proteins were quantified using a BCA kit (Beyotime, Shanghai, China) and were dissolved in loading buffer (Invitrogen). After electrophoresis on $10 \%(\mathrm{w} / \mathrm{v})$ sodium dodecyl sulfate-polyacrylamide (SDS) gels, proteins were transferred to polyvinylidenefluoride (PVDF) membranes (Millipore, Billerica, MA, USA) followed by incubation with $5 \%$ milk proteins in Tris-buffered saline containing $0.1 \%$ Triton $\mathrm{X}-100$ at $37^{\circ} \mathrm{C}$ for $1 \mathrm{~h}$. Next, the membrane was incubated with primary antibodies against GAPDH (1:1,000 dilution), matrix metalloproteinase 13 (MMP13, 1:500 dilution) and a disintegrin and metalloproteinase with thrombospondin motifs 5 (ADAMTS5, 1:500 dilution) at $4^{\circ} \mathrm{C}$ overnight. The mouse monoclonal anti-rat GAPDH (sc-47724) antibody, the rabbit polyclonal anti-rat MMP13 (sc-30073) and ADAMTS5 (sc-134952) antibodies were purchased from Santa Cruz Biotechnology, Inc. (Santa Cruz, CA, USA). The goat polyclonal anti-mouse $\operatorname{IgG}(\mathrm{H}+\mathrm{L})$ horseradish peroxidase (HRP)-conjugated secondary antibody and the goat anti-rabbit IgG $(\mathrm{H}+\mathrm{L}) \mathrm{HRP}$-conjugated secondary antibody (ZSGB-Bio, Beijing, China) were used. Immunolabeling was visualized using electrochemiluminescence (ECL) reagent (Thermo Fisher Scientific).

Cell growth assay. NP cells seeded in 96-well culture plates were incubated with $10 \mu \mathrm{l}$ of cell counting kit- 8 reagent (Dojindo, Tokyo, Japan) at $37^{\circ} \mathrm{C}$ for $2 \mathrm{~h}$. The absorbance was detected at $450 \mathrm{~nm}$ using a spectrophotometer (Varioskan Flash, Thermo Fisher Scientific) daily for 7 days. Then, absorbance data were used to calculate the number of NP cells based on the standard curve. Population doubling (PD) was calculated in following ways: $\mathrm{PD}=\left[\log _{10}(\mathrm{NH})-\log _{10}(\mathrm{NI})\right] / \log _{10}(2)$. $\mathrm{NH}$ is the calculated number of NP cells every day. NI is the initial number of NP cells seeded in the 96-well plate. PD values were used to depict the growth curve of NP cells.

Cell cycle analysis. NP cells were trypsinized and washed with PBS three times. To analyze the cell cycle distribution of NP cells, NP cells were resuspended in $1 \mathrm{ml}$ propidium iodide working solution of Cell Cycle Analysis kit (Beyotime). Then, the percentage of NP cells in different cell cycle phases including G1, G2 and S was measured by a flow cytometer (Beckman Coulter).

Statistical analysis. Independent experiments were performed at least three times. All results were presented as mean or mean \pm standard error of the mean. Two-tailed Student's t-test was used for the comparison between two independent groups. RT-qPCR results were analyzed using Kruskal-Wallis nonparametric analysis and Mann-Whitney U post-hoc tests. GraphPad Prism 6 (GraphPad Software Inc., La Jolla, CA, USA) and SPSS version 22.0 (International Business Machines Corp., Amonk, NY, USA) were used in this study. $\mathrm{P}<0.05$ was considered statistically significant.

\section{Results}

Analysis and functional annotation of DEGs induced by high oxygen tension. DEGs were identified according to the criteria mentioned above. There are 2499 DEGs in NP cells treated with high oxygen tension compared with the control. The percentage of upregulated genes $(1243 / 2499=49.7 \%)$ was nearly the same as the percentage of downregulated genes $(1256 / 2499=50.3 \%)$. To validate the results of the microarray, 13 genes were selected as the representative DEGs for RT-qPCR analysis. Eleven of the 13 representative genes showed the expression pattern that was consistent with the results of microarray (Fig. 1). The expression of MMP13, ADAMTS5, nicotinamide adenine dinucleotide phosphate (NADPH) oxidase 4 (Nox4), cyclin-dependent kinase inhibitor 2B (p15), bone morphogenetic protein 3 (BMP3), BMP4, growth arrest specific 1 (GAS1) and neuropilin 1 was significantly upregulated together with the markedly downregulation of vascular endothelial growth factor A (VEGFA), cyclin I and glutathione peroxidase 1 (GPX1).

GO analysis was performed to annotate the functions of DEGs. Many GO terms were related to the carbohydrate metabolism of NP cells, such as carbohydrate biosynthetic process, glycolytic process, gluconeogenesis and pyruvate metabolic process. Fig. 2A showed the top 10 biological responses enriched by DEGs in NP cells treated with high oxygen tension.

KEGG pathway analysis showed that various pathways were involved in the response of NP cells to high oxygen tension, including protein processing in endoplasmic reticulum, carbon metabolism, mTOR signaling pathway and PI3K-Akt signaling pathway. The top $10 \mathrm{KEGG}$ pathways enriched by DEGs are shown in Fig. 2B.

Analysis and functional annotation of ASGs induced by high oxygen tension. According to the filter criteria, 27004 ASEs derived from 8451 ASGs were identified. Among them, 10434 ASEs underwent general exon inclusion $(\mathrm{SI} \geq 2$ ). 16570 ASEs underwent general exon exclusion (SI $\leq-2$ ). $87.5 \%(23619 / 27004)$ of ASEs were derived from $54.6 \%$ (4616/8451) of ASGs, suggesting that a gene contains multiple ASEs in NP cells. For example, both aggrecan and ADAMTS5 had 7 ASEs, respectively. Furthermore, 1986 of the 8451 ASGs were differentially expressed. Semi-quantitative RT-PCR results of 10 representative genes are shown in Fig 3. Nine of the 10 genes had cassette alternative exons.

GO analysis showed that various biological processes were enriched by ASGs, including cellular macromolecule metabolic process, macromolecule modification, cell cycle, cellular response to oxidative stress, stress-activated MAPK cascade, angiogenesis, cell aging and regulation of autophagy. Fig. 4A showed the top 10 biological processes enriched by ASGs in NP cells treated with high oxygen tension. Moreover, several 

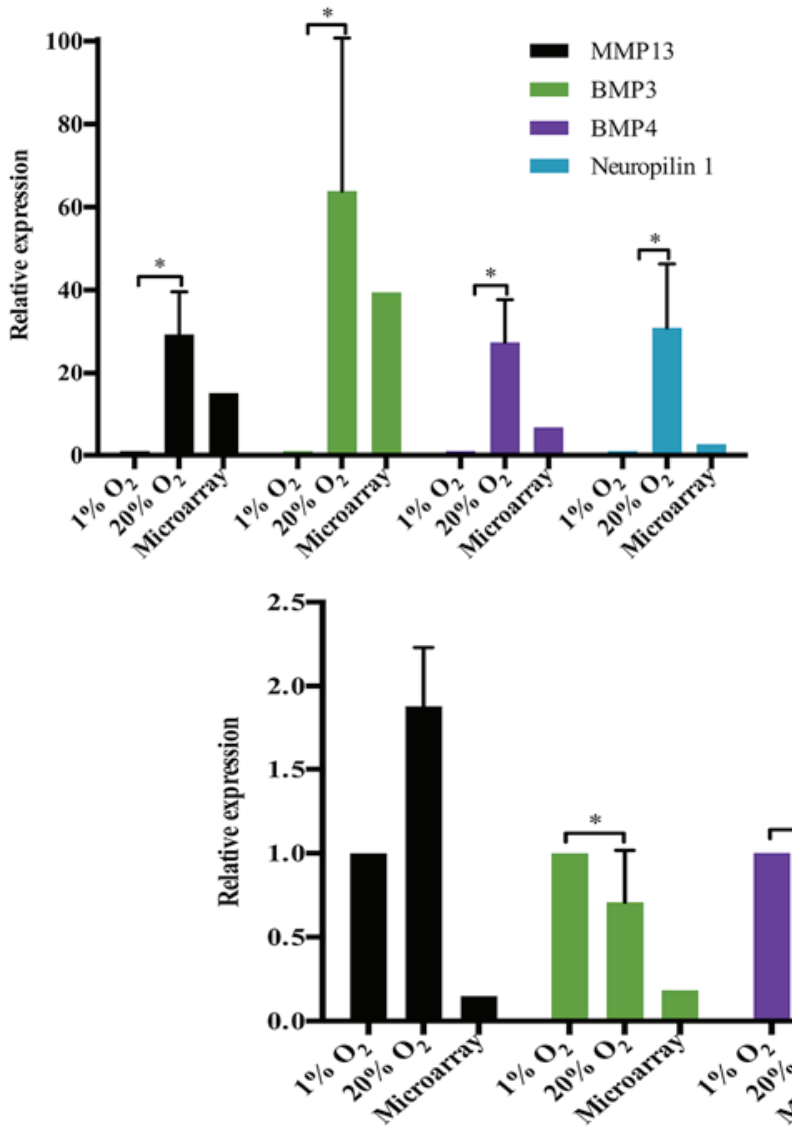
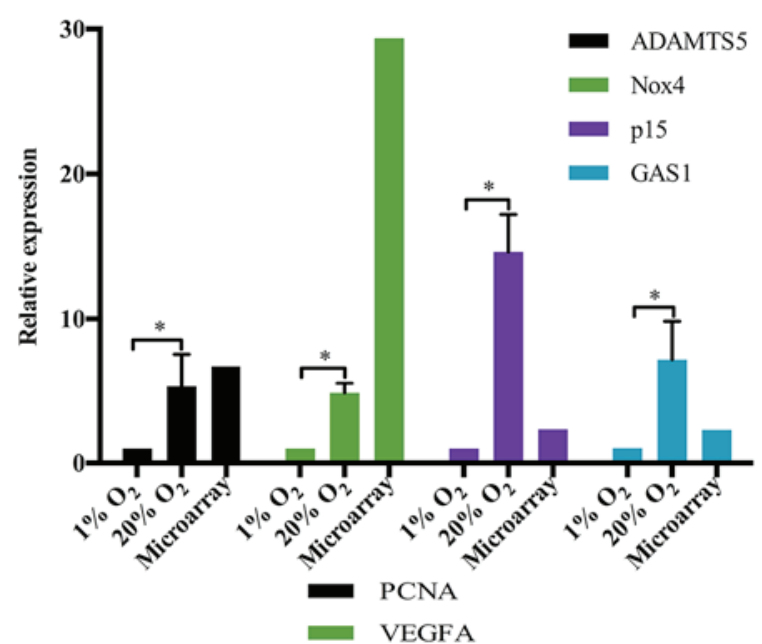

- Cyclin I

- GDF15

GPX1

Figure 1. Validation of the representative differentially expressed genes by RT-qPCR. "p-value $<0.05$, error bars represent standard error. MMP13, matrix metalloproteinase 13; ADAMTS5, a disintegrin and metalloproteinase with thrombospondin motifs 5; BMP, bone morphogenetic protein; Nox4, nicotinamide adenine dinucleotide phosphate (NADPH) oxidase 4; GAS1, growth arrest specific 1; PCNA, proliferating cell nuclear antigen; VEGFA, vascular endothelial growth factor A; GDF15, growth and differentiation factor 15; GPX1, glutathione peroxidase 1.

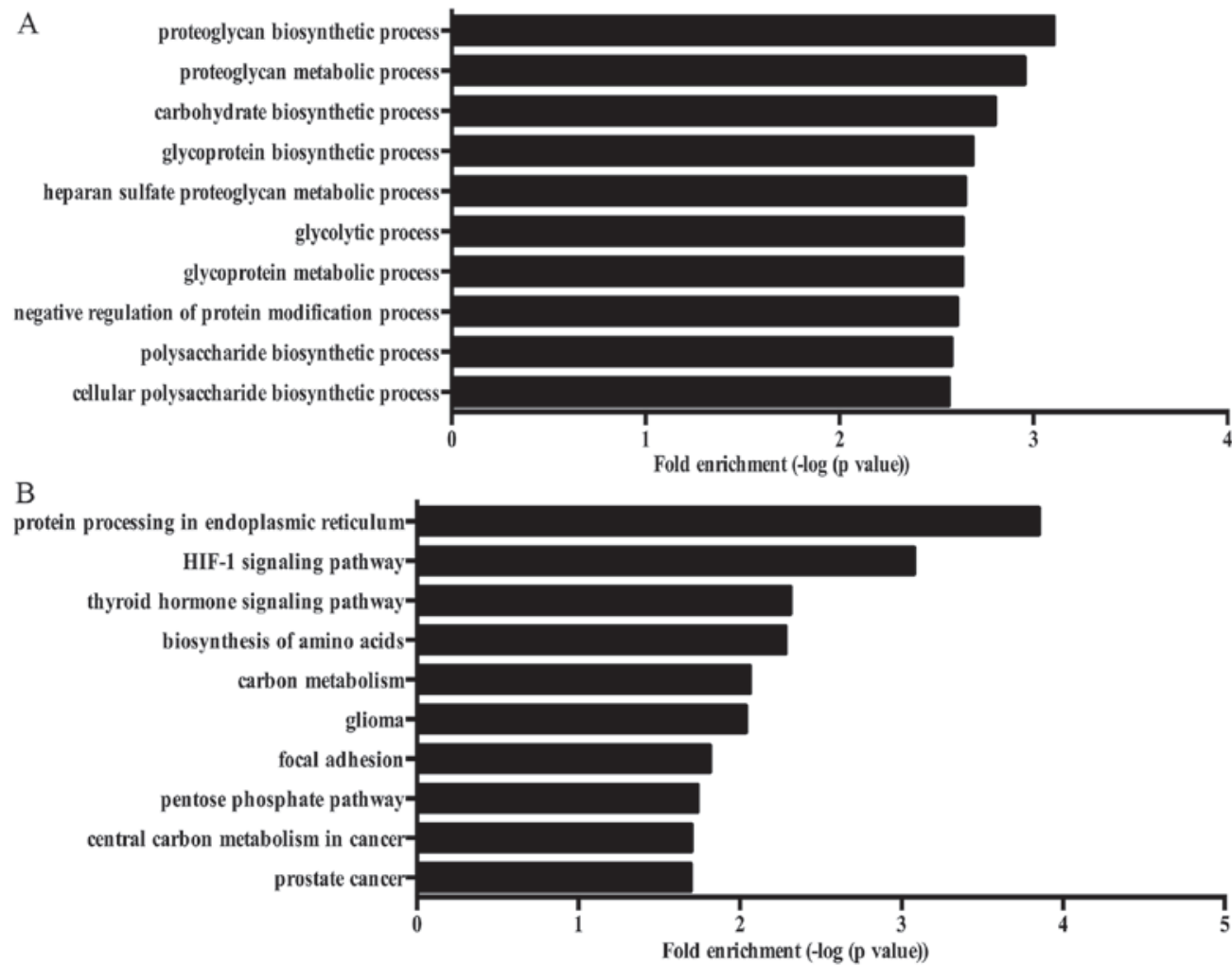

Figure 2. The Gene Ontology (GO) analysis and Kyoto Encyclopedia of Genes and Genomes (KEGG) pathway analysis of differentially expressed genes. (A) Top 10 biological processes enriched by differentially expressed genes. (B) Top 10 KEGG pathways enriched by differentially expressed genes. 


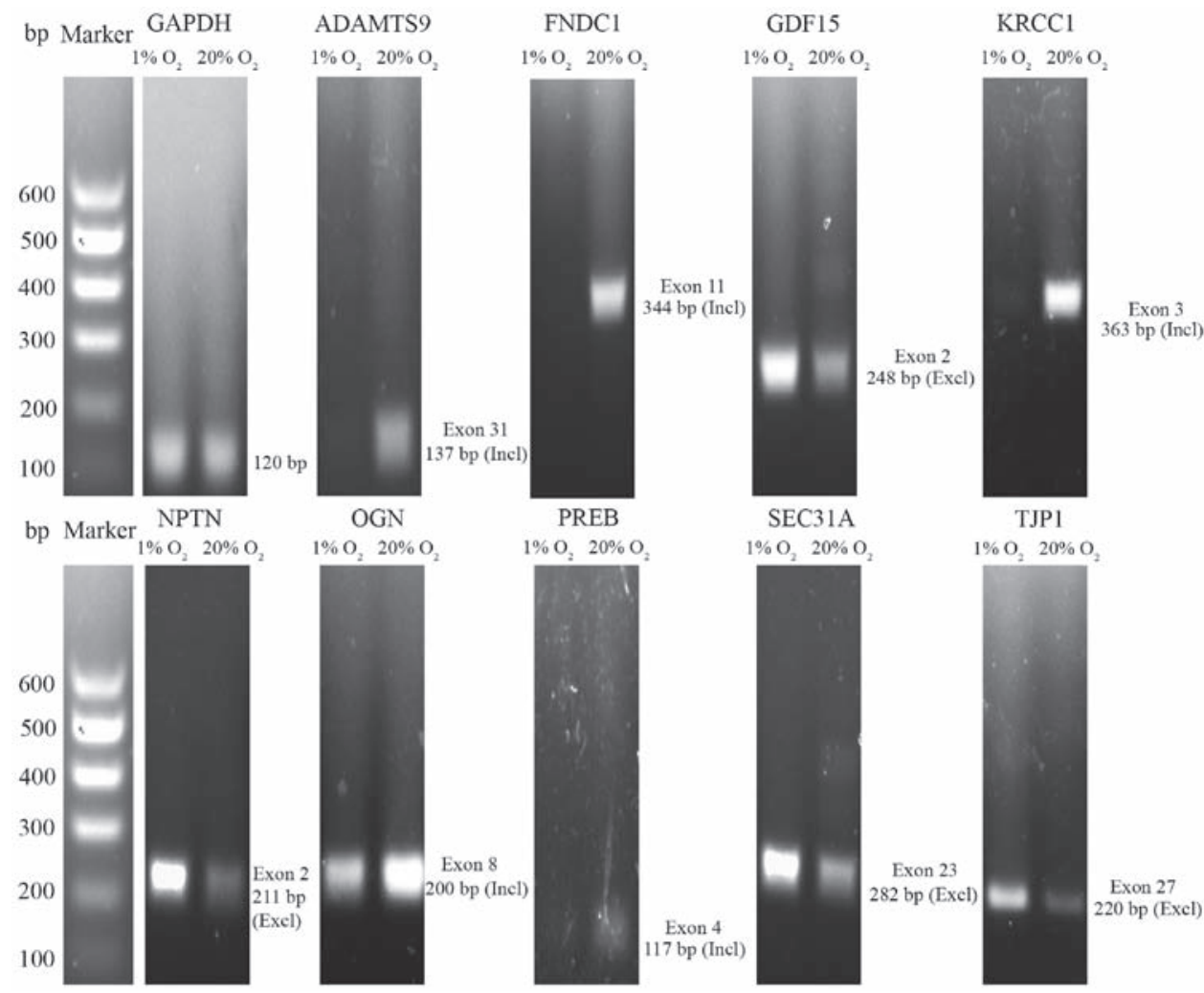

Figure 3. Validation of the representative alternative splicing genes by semi-quantitative RT-PCR. ADAMTS9, a disintegrin and metalloproteinase with thrombospondin motifs 9; FNDC1, fibronectin type III domain containing 1; GDF15, growth and differentiation factor 15; KRCC1, lysine-rich coiledcoil 1; NPTN, neuroplastin; OGN, osteoglycin; PREB, prolactin regulatory element binding; SEC31A, SEC31 homolog A, COPII coat complex component; TJP1, tight junction protein 1; Incl, inclusion; Excl, exclusion.

A

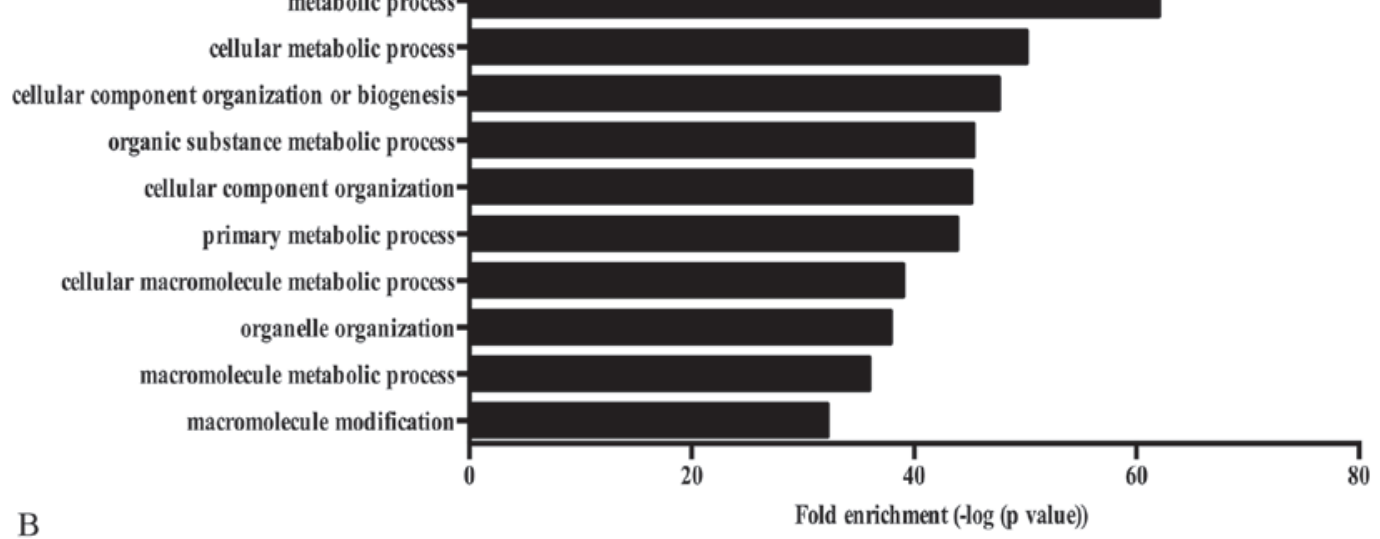

B

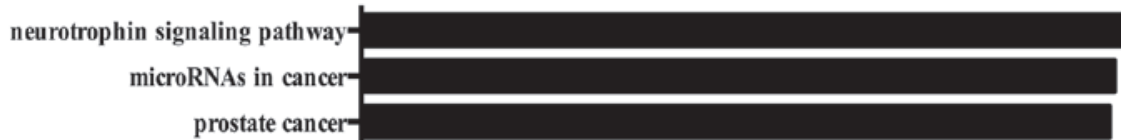

ErbB signaling pathway-

pancreatic cancer-

chronic myeloid leukemia-

acute myeloid leukemia-

phosphatidylinositol signaling system-

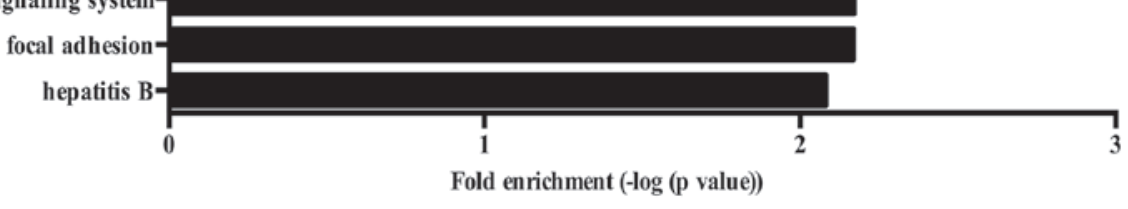

Figure 4. The Gene Ontology (GO) analysis and Kyoto Encyclopedia of Genes and Genomes (KEGG) pathway analysis of alternative splicing genes. (A) Top 10 biological processes enriched by alternative splicing genes. (B) Top 10 KEGG pathways enriched by alternative splicing genes. 

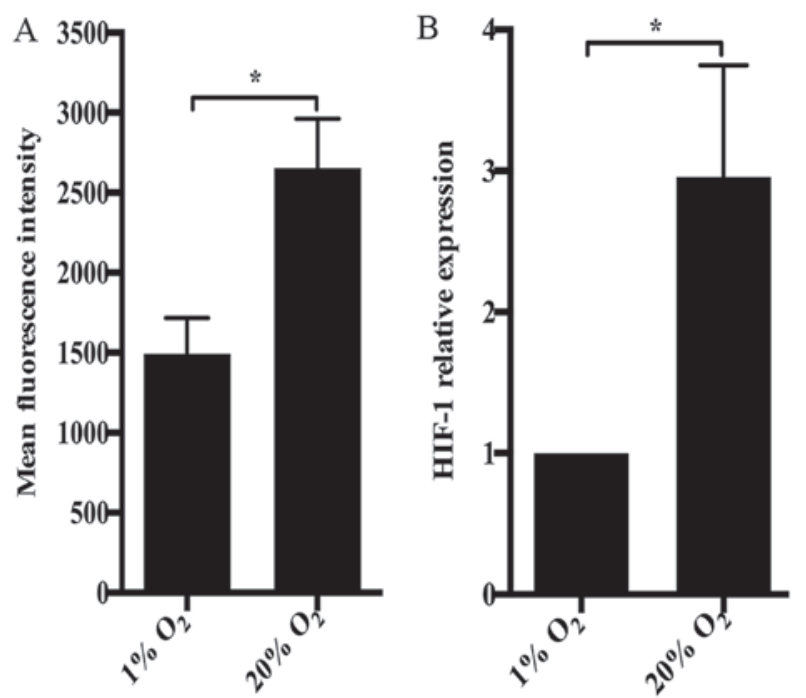

Figure 5. High oxygen tension increases ROS production and expression of hypoxia inducible factor-1 (HIF-1) in nucleus pulposus (NP) cells. (A) ROS production in high oxygen tension-treated NP cells. (B) Quantitative PCR analysis of HIF-1 in high oxygen tension-treated NP cells. ${ }^{*}$ p-value $<0.05$, error bars represent standard error.

KEGG pathways associated with pathogenesis of IDD were enriched by ASGs, such as neurotrophin signaling pathway and mTOR signaling pathway. Fig. 4B shows the top $10 \mathrm{KEGG}$ pathway enriched by ASGs.

High oxygen tension increases ROS generation in NP cells. Several biological responses enriched by DEGs or ASGs were correlated with oxygen tension, such as cellular response to oxygen levels and cellular response to hypoxia. Also, biological responses related to redox homeostasis, including positive regulation of ROS metabolic process, glutathione metabolic process and cellular response to ROS, were identified. In fact, ROS production was dramatically increased by high oxygen tension in NP cells (Fig. 5A). It was consistent with the upregulation of Nox4 and the downregulation of GPX1 in NP cells treated with high oxygen tension (Fig. 1). Notably, hypoxia inducible factor-1 (HIF-1) signaling pathway was enriched by DEGs and ASGs. Therefore, we investigated the expression of HIF-1 in NP cells using RT-qPCR. Paradoxically, the expression of HIF-1 in NP cells subjected to $20 \% \mathrm{O}_{2}$ was significantly higher than that in NP cells under $1 \% \mathrm{O}_{2}$ (Fig. 5B).

Effect of high oxygen tension on matrix metabolism of NP cells. GO terms and KEGG pathways associated with matrix metabolism of disc cells have been shown to be enriched by DEGs, including proteoglycan metabolic process, tissue remodeling and glycosaminoglycan biosynthesis. Thus, we investigated the expression of collagen type II and aggrecan in NP cells. We found that collagen type II and aggrecan were markedly upregulated by high oxygen tension (Fig. 6A). However, two crucial matrix degradation proteases in IVDs, MMP13 and ADAMTS5 also were upregulated by high oxygen tension in NP cells (Fig. 6B).

High oxygen tension arrested the G1/S transition of NP cell cycle. The proliferation of disc cells is crucial to the structural and functional maintenance of IVDs. The proliferation of NP cells depends on cell cycle progression. Interestingly, various GO terms and KEGG pathways enriched by DEGs or ASGs were associated with the cell cycle of NP cells, including cell cycle arrest, G1/S phase transition of cell cycle, regulation of cell growth, mitotic G1/S transition checkpoint and regulation of cyclin-dependent protein serine/threonine kinase activity involved in G1/S transition of mitotic cell cycle. Actually, the upregulation of p15 and GAS1 together with the downregulation of cyclin I in NP cells treated with high oxygen tension have been mentioned (Fig. 1). At the same time, the G1/S phase transition of NP cell cycle was retarded by high oxygen tension (Fig. 7A). As a result, the growth of NP cells was suppressed by high oxygen tension (Fig. 7B). On the other hand, several GO terms, such as DNA damage response, G1 DNA damage checkpoint, signal transduction by p53 class mediator resulting in transcription of p21 class mediator and p53 signaling pathway, indicate that the cell cycle arrest of NP cells induced by high oxygen tension is potently associated with DNA damage checkpoint in G1/S transition.

\section{Discussion}

The viability and function of disc cells are essential to the homeostasis maintenance system of IVDs (36). Investigating the effect of high oxygen tension on disc cells contributes to elucidating the role of high oxygen tension in the pathogenesis of IDD. Exposure of NP cells to high oxygen tension has been demonstrated as an effective method to increase ROS production and consequently cause oxidative stress in NP cells. Excessive ROS generation induced by high oxygen tension has been shown to reinforce the matrix catabolism and autophagy of NP cells $(21,22)$. However, to our knowledge, there were no studies that analyze the gene expression profile of disc cells in response to high oxygen tension on a genome-wide scale. Moreover, the involvement of AS in regulating the response of disc cells to high oxygen tension remains unknown. Therefore, this study, for the first time, performed a genome-wide analysis to determine the global gene expression profile and AS in NP cells subjected to high oxygen tension. More than two thousand genes were significantly differentially expressed in NP cells subjected to high oxygen tension. The results of bioinformatics analysis revealed a wide role of high oxygen tension in regulating the viability and function of disc cells.

The involvement of AS in various diseases has aroused the interest of many studies. However, few studies investigated the role of AS in the pathogenesis of IDD. Our study provides a genome-wide view to analyze ASGs in NP cells treated with high oxygen tension, and 27004 ASEs derived from 8451 ASGs were determined. Nine of the 10 representative ASGs were confirmed by semi-quantitative RT-PCR. The results suggest the effect of high oxygen tension on the AS of NP cells. Among the 10 representative ASGs, ADAMTS9 is an aggrecanase, and growth and differentiation factor 15 is a member of TGF superfamily. Matrix proteases and growth factors have been demonstrated to be strongly associated with IDD (37,38). Furthermore, numerous GO terms and KEGG pathways enriched by ASGs were correlated with the pathogenesis of IDD, including regulation of autophagy, mTOR signaling pathway and neurotrophin signaling pathway. 


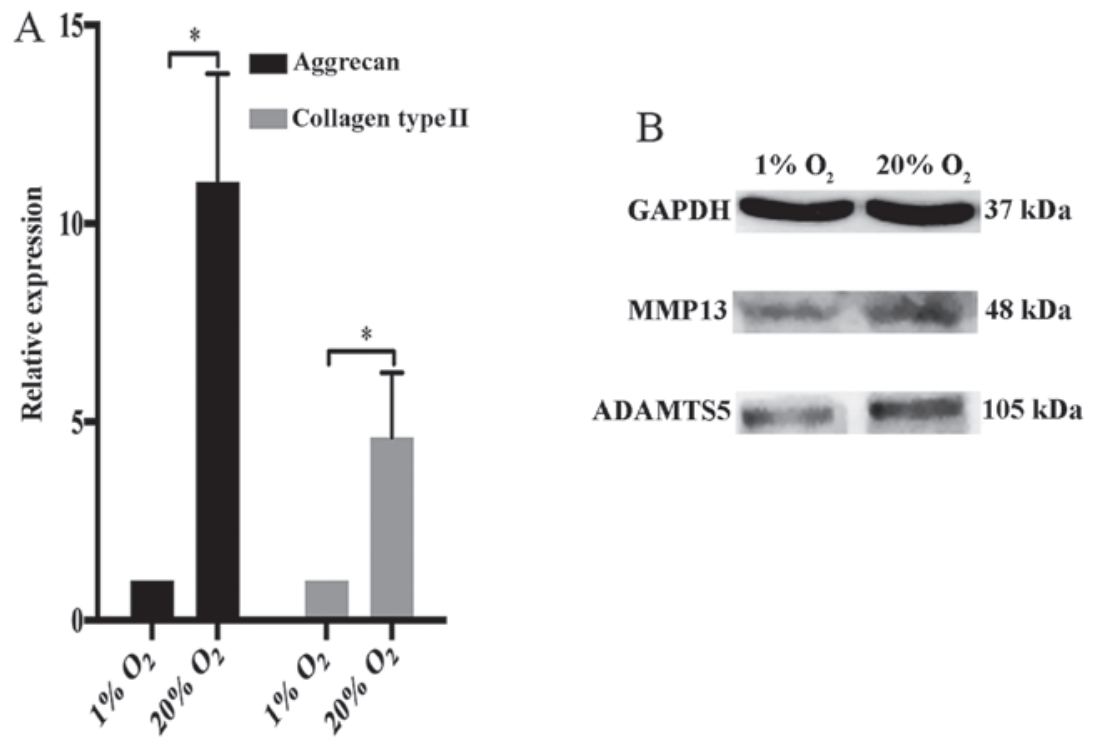

Figure 6. High oxygen tension accelerats both matrix anabolism and catabolism in nucleus pulposus (NP) cells. (A) Quantitative PCR analysis of aggrecan and collagen type II in high oxygen tension-treated NP cells. (B) Immunoblot analysis of matrix metalloproteinase 13 (MMP13) and a disintegrin and metalloproteinase with thrombospondin motifs 5 (ADAMTS5) in high oxygen tension-treated NP cells. "P-value $<0.05$, error bars represent standard error.
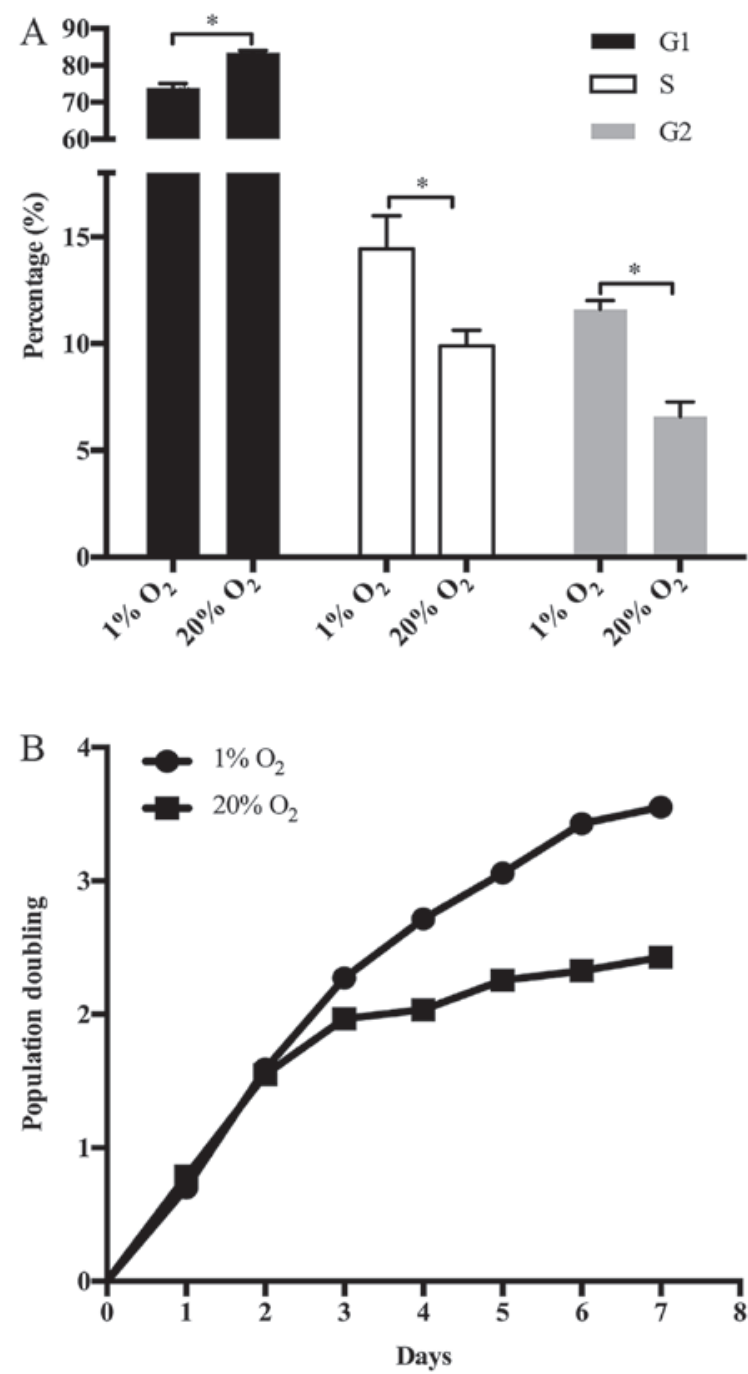

Figure 7. High oxygen tension arrests the cell cycle progression of nucleus pulposus (NP) cells from G1 to S phase, and retards the growth of NP cells. (A) Cell cycle distribution of NP cells. (B) The growth curve of NP cells. * $\mathrm{p}$-value $<0.05$, error bars represent standard error.
Disc cell autophagy has been demonstrated to be an essential event in the process of IDD. It is a double-edged sword. The appropriate autophagy promotes the survival of disc cells through self-digestion that protects disc cells from various stresses. However, excessive autophagy causes disc cell death to decrease the number of viable and functional cells in discs $(20,22,39)$. With regard to the mechanism underling disc cell autophagy, mTOR pathway is a crucial regulatory signaling pathway $(40,41)$. Besides, neurotrophin is responsible for neuronal ingrowth in degenerative discs that contributes to pain sensation in LBP $(42,43)$. In short, AS induced by high oxygen tension is widely involved in regulating the biological process and signaling pathways of disc cells and consequently affects the initiation and progression of IDD.

BMP4 has been reported to be associated with IDD (44). The possible involvement of BMP4 and its receptor in the chondrogenic progress of spondylosis also has been mentioned (45). However, the precious role of BMP4 in the pathogenesis of IDD remains unclear. Another member of the BMP family, BMP3, has not been investigated in IVDs so far. Herein, both BMP3 and BMP4 were dramatically upregulated by high oxygen tension in NP cells. The GO term, regulation of BMP signaling pathway, was enriched by both DEGs and ASGs, suggesting that BMP3 and BMP4 are involved in regulating the responses of NP cells to high oxygen tension. Revealing the relationship between BMP and high oxygen tension extends our knowledge on the role of BMP in the pathogenesis of IDD.

Enhanced blood vessel ingrowth into NP is a crucial pathological characteristic of degenerative discs. Neovascularization leads to the exposure of disc cells to higher oxygen tension. VEGF, an angiogenic factor, has been demonstrated to induce neovascularization in herniated discs $(43,46)$. NP cells upregulate the expression of VEGF in response to mechanical strain and pro-inflammatory cytokines, which enhances angiogenesis in IVDs $(47,48)$. On the contrary, herein, we found that the expression of VEGF in NP cells was downregulated by high 
oxygen tension, suggesting that high oxygen tension suppresses angiogenesis in discs, probably forming a negative feedback. Therefore, the effect of high oxygen tension on neovascularization in degenerative discs should be assessed in further studies.

Consistent with previous studies $(21,22), 20 \% \mathrm{O}_{2}$ significantly increased ROS levels in NP cells. The GO terms enriched by DEGs or ASGs, positive regulation of ROS metabolic process, cellular response to ROS and glutathione metabolic process, suggest that oxygen tension regulates the balance between antioxidant generation and ROS production in NP cells. The differential expression of Nox4 and GPX1 supports this idea further. Nox4 is a professional ROS-generating enzyme and a major source of intracellular ROS (49). The upregulation of Nox4 induced by high oxygen tension can explain increased ROS production in NP cells subjected to high oxygen tension. On the other hand, GPX1, a major antioxidant gene, was markedly downregulated by high oxygen tension, indicating a decline in antioxidants of NP cells. In conclusion, high oxygen tension disturbs the redox homeostasis to arouse oxidative stress in NP cells. Besides, the expression of HIF-1 was paradoxically upregulated in NP cells exposed to high oxygen tension. This issue should be investigated further in depth.

The structural and functional maintenance of IVDs depends on the balance between the ECM anabolism and catabolism of discs. Noticeably, high oxygen tension not only upregulated the expression of aggrecan and collagen type II, but also upregulated the expression of MMP13 and ADAMTS5 in NP cells. High oxygen tension showed both anabolic and catabolic effects on the matrix metabolism of NP cells. Thus, we hypothesize that high oxygen tension accelerates matrix turnover of IVDs. Nevertheless, we still need more in vivo evidence to elucidate the comprehensive effect of high oxygen tension on matrix homeostasis of IVDs.

The proliferation of disc cells is crucial to maintaining the number of functional cells in IVDs. It relies on cell cycle progression of disc cells. In this study, as the GO terms, cell cycle arrest and G1/S phase transition of cell cycle, suggested, high oxygen tension regulated the expression of p15, GAS1 and cyclin I, and subsequently retarded the G1/S phase transition of NP cell cycle. As a consequence, the growth of NP cells was arrested. The detrimental effect of high oxygen tension on NP cell proliferation indicates that high oxygen tension may cause a decrease in the number of functional and viable cells in discs. Mechanistically, DNA damage is a widely known intrinsic trigger of cell cycle arrest. It activates the G1/S cell cycle checkpoint to retard cell cycle progression. Furthermore, ROS have been demonstrated as potent genotoxic agents $(17,50)$. Therefore, it can be speculated that oxidative stress induced by high oxygen tension enhances DNA damage to arrest the cell cycle progression of NP cells from G1 to S phase.

There are several limitations in the current study. One limitation is that we investigated the effect of high oxygen tension on the global gene expression profile and AS of rat NP cells. Further studies based on human disc cells should be performed. On the other hand, this study elucidated the involvement of high oxygen tension in the establishment and progression of IDD sketchily. The precise roles of high oxygen tension in regulating the viability and function of disc cells and the pathogenesis of IDD are required to be discussed in detail.
In conclusion, high oxygen tension is widely involved in regulating various biological processes of NP cells through transcription regulation and AS regulation. Several processes are potently associated with the process of IDD. Specifically, it disturbs the redox homeostasis of NP cells and promotes matrix turnover in discs. Furthermore, high oxygen tension retards NP cell cycle progression from G1 to S phase, which consequently suppresses the growth of NP cells. High oxygen tension is a crucial driver to the disc cell-mediated IDD process.

\section{Acknowledgements}

We thank Dr Yi Zha for his help in analyzing the microarray data.

\section{Funding}

The design of the study and collection, analysis, and interpretation of data and writing of the manuscript study were supported by the National Natural Science Foundation of China (grant nos. 81672215, 81572186, 81271982, 81472076 and 81401801).

\section{Availability of data and material}

All data generated or analyzed during this study are included in this published article.

\section{Authors' contributions}

CF contributed to the conception and design, acquisition of data, analysis and interpretation of data, and manuscript writing. YaZ contributed to the acquisition of data and provision of study material or patients. MY and ML contributed to the acquisition of data and analysis and interpretation of data. $\mathrm{BH}$ contributed to the conception and design and final approval of the version to be published. HL gave the final approval of the version to be published, and contributed to the conception and design, financial and administrative support. YuZ gave the final approval of the version to be published, and contributed to the conception and design, financial and administrative support. All authors read and approved the final manuscript.

\section{Ethics approval and consent to participate}

This research was approved by the Ethics Committee of Xinqiao Hospital. All procedures described in this study were in accordance with the standards set forth in the eighth edition of the Guide for the Care and Use of Laboratory Animals published by the National Academy of Sciences (Washington, DC, USA).

\section{Consent for publication}

Not applicable.

\section{Competing interests}

The authors declare that they have no competing interests. 


\section{References}

1. Vos T, Flaxman AD, Naghavi M,Lozano R, Michaud C,Ezzati M Shibuya K, Salomon JA, Abdalla S, Aboyans V, et al: Years lived with disability (YLDs) for 1160 sequelae of 289 diseases and injuries 1990-2010: A systematic analysis for the Global Burden of Disease Study 2010. Lancet 380: 2163-2196, 2012.

2. Hong J, Reed C, Novick D and Happich M: Costs associated with treatment of chronic low back pain: An analysis of the UK General Practice Research Database. Spine 38: 75-82, 2013.

3. Roberts S, Evans H, Trivedi J and Menage J: Histology and pathology of the human intervertebral disc. J Bone Joint Surg Am 88 (Suppl 2): 10-14, 2006.

4. Battié MC, Videman T, Kaprio J, Gibbons LE, Gill K, Manninen H, Saarela J and Peltonen L: The Twin Spine Study: Contributions to a changing view of disc degeneration. Spine J 9: 47-59, 2009.

5. Xing QJ, Liang QQ, Bian Q, Ding DF, Cui XJ, Shi Q and Wang YJ: Leg amputation accelerates senescence of rat lumbar intervertebral discs. Spine 35: E1253-E1261, 2010.

6. Wang D, Nasto LA, Roughley P, Leme AS, Houghton AM, Usas A, Sowa G, Lee J, Niedernhofer L, Shapiro S, et al: Spine degeneration in a murine model of chronic human tobacco smokers. Osteoarthritis Cartilage 20: 896-905, 2012.

7. Stirling A, Worthington T, Rafiq M, Lambert PA and Elliott TS: Association between sciatica and Propionibacterium acnes. Lancet 357: 2024-2025, 2001.

8. Park EY and Park JB: Dose- and time-dependent effect of high glucose concentration on viability of notochordal cells and expression of matrix degrading and fibrotic enzymes. Int Orthop 37: 1179-1186, 2013.

9. Bartels EM, Fairbank JC, Winlove CP and Urban JP: Oxygen and lactate concentrations measured in vivo in the intervertebral discs of patients with scoliosis and back pain. Spine 23: 1-7, discussion 8, 1998.

10. Urban JP, Smith S and Fairbank JC: Nutrition of the intervertebral disc. Spine 29: 2700-2709, 2004.

11. Lee DC, Adams CS, Albert TJ, Shapiro IM, Evans SM and Koch CJ: In situ oxygen utilization in the rat intervertebral disc. J Anat 210: 294-303, 2007.

12. Risbud MV, Guttapalli A, Stokes DG, Hawkins D, Danielson KG, Schaer TP, Albert TJ and Shapiro IM: Nucleus pulposus cells express HIF-1 alpha under normoxic culture conditions: A metabolic adaptation to the intervertebral disc microenvironment. J Cell Biochem 98: 152-159, 2006.

13. Agrawal A, Guttapalli A, Narayan S, Albert TJ, Shapiro IM and Risbud MV: Normoxic stabilization of HIF-1alpha drives glycolytic metabolism and regulates aggrecan gene expression in nucleus pulposus cells of the rat intervertebral disk. Am J Physiol Cell Physiol 293: C621-C631, 2007.

14. Ali R, Le Maitre CL, Richardson SM, Hoyland JA and Freemont AJ: Connective tissue growth factor expression in human intervertebral disc: implications for angiogenesis in intervertebral disc degeneration. Biotech Histochem 83: 239-245, 2008.

15. Furusawa N, Baba H, Miyoshi N, Maezawa Y, Uchida K, Kokubo Y and Fukuda M: Herniation of cervical intervertebral disc: Immunohistochemical examination and measurement of nitric oxide production. Spine 26: 1110-1116, 2001

16. Suzuki S, Fujita N, Hosogane N, Watanabe K, Ishii K, Toyama Y, Takubo K, Horiuchi K, Miyamoto T, Nakamura M, et al: Excessive reactive oxygen species are therapeutic targets for intervertebral disc degeneration. Arthritis Res Ther 17: 316, 2015

17. Dimozi A, Mavrogonatou E, Sklirou A and Kletsas D: Oxidative stress inhibits the proliferation, induces premature senescence and promotes a catabolic phenotype in human nucleus pulposus intervertebral disc cells. Eur Cell Mater 30: 89-103, 2015.

18. Hou G, Lu H, Chen M, Yao H and Zhao H: Oxidative stress participates in age-related changes in rat lumbar intervertebral discs. Arch Gerontol Geriatr 59: 665-669, 2014.

19. Adams MA and Roughley PJ: What is intervertebral disc degeneration, and what causes it? Spine 31: 2151-2161, 2006.

20. Ding F, Shao ZW and Xiong LM: Cell death in intervertebral disc degeneration. Apoptosis 18: 777-785, 2013.

21. Nasto LA, Robinson AR, Ngo K, Clauson CL, Dong Q, St Croix C, Sowa G, Pola E, Robbins PD, Kang J, et al: Mitochondrialderived reactive oxygen species (ROS) play a causal role in aging-related intervertebral disc degeneration. J Orthop Res 31: $1150-1157,2013$
22. Chen JW, Ni BB, Zheng XF, Li B, Jiang SD and Jiang LS: Hypoxia facilitates the survival of nucleus pulposus cells in serum deprivation by downregulating excessive autophagy through restricting ROS generation. Int J Biochem Cell Biol 59: $1-10,2015$.

23. Pan Q, Shai O, Lee LJ, Frey BJ and Blencowe BJ: Deep surveying of alternative splicing complexity in the human transcriptome by high-throughput sequencing. Nat Genet 40: 1413-1415, 2008.

24. Black DL: Mechanisms of alternative pre-messenger RNA splicing. Annu Rev Biochem 72: 291-336, 2003.

25. Blencowe BJ: Alternative splicing: New insights from global analyses. Cell 126: 37-47, 2006.

26. Lukong KE, Chang KW, Khandjian EW and Richard S: RNA-binding proteins in human genetic disease. Trends Genet 24: 416-425, 2008

27. Martinez-Contreras R, Cloutier P, Shkreta L, Fisette JF, Revil T and Chabot B: hnRNP proteins and splicing control. Adv Exp Med Biol 623: 123-147, 2007.

28. Ng B, Yang F, Huston DP, Yan Y, Yang Y, Xiong Z, Peterson LE, Wang $\mathrm{H}$ and Yang XF: Increased noncanonical splicing of autoantigen transcripts provides the structural basis for expression of untolerized epitopes. J Allergy Clin Immunol 114: 1463-1470, 2004.

29. Anderson DG, Markova D, Adams SL, Pacifici M, An HS and Zhang Y: Fibronectin splicing variants in human intervertebral disc and association with disc degeneration. Spine 35: 1581-1588, 2010.

30. Hirose Y, Chiba K, Karasugi T, Nakajima M, Kawaguchi Y, Mikami Y, Furuichi T, Mio F, Miyake A, Miyamoto T, et al: A functional polymorphism in THBS2 that affects alternative splicing and MMP binding is associated with lumbar-disc herniation. Am J Hum Genet 82: 1122-1129, 2008.

31. Sztrolovics R, Grover J, Cs-Szabo G, Shi SL, Zhang Y, Mort JS Roughley PJ: The characterization of versican and its message in human articular cartilage and intervertebral disc. J Orthop Res 20: 257-266, 2002.

32. Purdom E, Simpson KM, Robinson MD, Conboy JG, Lapuk AV and Speed TP: FIRMA: A method for detection of alternative splicing from exon array data. Bioinformatics 24: 1707-1714, 2008.

33. Srinivasan K, Shiue L, Hayes JD, Centers R, Fitzwater S, Loewen R, Edmondson LR, Bryant J, Smith $M$ and Rommelfanger C: Detection and measurement of alternative splicing using splicing-sensitive microarrays. Methods 37: 345-359, 2005.

34. Clark TA, Sugnet CW and Ares M Jr: Genomewide analysis of mRNA processing in yeast using splicing-specific microarrays. Science 296: 907-910, 2002.

35. Livak KJ and Schmittgen TD: Analysis of relative gene expression data using real-time quantitative PCR and the 2(-Delta Delta C(T)) method. Methods 25: 402-408, 2001.

36. Sakai D and Andersson GB: Stem cell therapy for intervertebral disc regeneration: Obstacles and solutions. Nat Rev Rheumatol 11: 243-256, 2015.

37. Vo NV, Hartman RA, Yurube T, Jacobs LJ, Sowa GA and Kang JD: Expression and regulation of metalloproteinases and their inhibitors in intervertebral disc aging and degeneration. Spine J 13: 331-341, 2013.

38. Feng C, Liu H, Yang Y, Huang B and Zhou Y: Growth and differentiation factor-5 contributes to the structural and functional maintenance of the intervertebral disc. Cell Physiol Biochem 35: 1-16, 2015.

39. Shen C, Yan J, Jiang LS and Dai LY: Autophagy in rat annulus fibrosus cells: Evidence and possible implications. Arthritis Res Ther 13: R132, 2011.

40. Chen JW, Ni BB, Li B, Yang YH, Jiang SD and Jiang LS: The responses of autophagy and apoptosis to oxidative stress in nucleus pulposus cells: implications for disc degeneration. Cell Physiol Biochem 34: 1175-1189, 2014.

41. Jiang L, Jin Y, Wang H, Jiang Y and Dong J: Glucosamine protects nucleus pulposus cells and induces autophagy via the mTOR-dependent pathway. J Orthop Res 32: 1532-1542, 2014.

42. Binch AL, Cole AA, Breakwell LM, Michael AL, Chiverton N, Creemers LB, Cross AK and Le Maitre CL: Nerves are more abundant than blood vessels in the degenerate human intervertebral disc. Arthritis Res Ther 17: 370, 2015.

43. Binch AL, Cole AA, Breakwell LM, Michael AL, Chiverton N, Cross AK and Le Maitre CL: Expression and regulation of neurotrophic and angiogenic factors during human intervertebral disc degeneration. Arthritis Res Ther 16: 416, 2014. 
44. Takae R, Matsunaga S, Origuchi N, Yamamoto T, Morimoto N, Suzuki S and Sakou T: Immunolocalization of bone morphogenetic protein and its receptors in degeneration of intervertebral disc. Spine 24: 1397-1401, 1999.

45. Nakase T, Ariga K, Miyamoto S, Okuda S, Tomita T, Iwasaki M, Yonenobu $\mathrm{K}$ and Yoshikawa $\mathrm{H}$ : Distribution of genes for bone morphogenetic protein-4, -6 , growth differentiation factor-5, and bone morphogenetic protein receptors in the process of experimental spondylosis in mice. J Neurosurg 94 (Suppl 1): 68-75, 2001.

46. Haro H, Kato T, Komori H, Osada M and Shinomiya K: Vascular endothelial growth factor (VEGF)-induced angiogenesis in herniated disc resorption. J Orthop Res 20: 409-415, 2002.

47. Gawri R, Rosenzweig DH, Krock E, Ouellet JA, Stone LS, Quinn TM and Haglund L: High mechanical strain of primary intervertebral disc cells promotes secretion of inflammatory factors associated with disc degeneration and pain. Arthritis Res Ther 16: R21, 2014.
48. Lee JM, Song JY, Baek M, Jung HY, Kang H, Han IB, Kwon YD and Shin DE: Interleukin-1 $\beta$ induces angiogenesis and innervation in human intervertebral disc degeneration. J Orthop Res 29: 265-269, 2011.

49. Sahoo S, Meijles DN and Pagano PJ: NADPH oxidases: key modulators in aging and age-related cardiovascular diseases? Clin Sci (Lond) 130: 317-335, 2016.

50. Weyemi U, Lagente-Chevallier O, Boufraqech M, Prenois F, Courtin F, Caillou B, Talbot M, Dardalhon M, Al Ghuzlan A, Bidart JM, et al: ROS-generating NADPH oxidase NOX4 is a critical mediator in oncogenic H-Ras-induced DNA damage and subsequent senescence. Oncogene 31: 1117-1129, 2012.

(i)(9) This work is licensed under a Creative Commons Attribution-NonCommercial-NoDerivatives 4.0 International (CC BY-NC-ND 4.0) License. 